\title{
LOCALLY UNIVALENT FUNCTIONS WITH LOCALLY UNIVALENT DERIVATIVES $\left({ }^{1}\right)$
}

\author{
BY \\ DOUGLAS MICHAEL CAMPBELL
}

\begin{abstract}
S. M. Shah and S. Y. Trimble have discovered that the behavior of an analytic function $f(z)$ is strongly influenced by the radii of univalence of its derivatives $f^{(n)}(z)(n=0,1,2, \ldots)$. In this paper many of Shah and Trimble's results are extended to large classes of locally univalent functions with locally univalent derivatives. The work depends on the concept of the $\mathscr{U}_{\beta}$-radius of a locally univalent function that is introduced and developed in this paper. Ch. Pommerenke's definition of a linear invariant family of locally univalent functions and the techniques of that theory are employed in this paper. It is proved that the universal linear invariant families $\mathscr{U}_{\alpha}$ are rotationally invariant. For fixed $f(z)$ in $\mathscr{U}_{\alpha}$, it is shown that the function $r \rightarrow \operatorname{order}[f(r z) / r](0<r \leqq 1)$ is a continuous increasing function of $r$.
\end{abstract}

1. Introduction. In a recent series of papers [8], [9], [10], Shah and Trimble have investigated the properties of a function that is analytic in the unit disc and for which the radii of univalence of the derivatives $f^{(n)}(z)(n=0,1,2, \ldots)$ are restricted in various ways. In this paper we generalize many of their results by replacing univalence with local univalence and replacing the radius of univalence with the $\mathscr{U}_{\beta}$-radius (a quantity explained in $\S 4$ ). The concept of $\mathscr{U}_{\beta}$-radius is more general than that of radius of univalence. In general it is very difficult to compute the radius of univalence of a function. However, in many instances we can compute the $\mathscr{U}_{\beta}$-radius when we cannot determine the radius of univalence. The notion of $\mathscr{U}_{\beta}$-radius stems from the concept of the universal linear invariant families of order $\alpha$ which were first introduced by Pommerenke [6]. This paper is a continuation of the theme that many theorems concerning univalence find a better setting in the context of linear invariant families of locally univalent functions [2], [6].

For each universal linear invariant family $\mathscr{U}_{\alpha}$ the quantity

$$
\alpha=\sup \left\{\left|f^{\prime \prime}(0) / 2\right|: f \in \mathscr{U}_{\alpha}\right\}
$$

is very important [6]. In Theorem 2 we obtain an asymptotic estimate for $\sup \left|f^{\prime \prime}(0) / 2\right|$ when additional restrictions are placed on all of the derivatives

Presented to the Society, January 22, 1971; received by the editors September 17, 1970 and, in revised form, April 15, 1971.

AMS 1969 subject classifications. Primary 3042, 3055; Secondary 3044, 3052.

Key words and phrases. Analytic function, univalent function, entire function, linear invariant family of locally univalent functions, order of a family, admissible property, the $\mathscr{U}_{\beta}$-radius, rotational invariance, contractional invariance.

( ${ }^{1}$ ) This research was supported by the U.S. Army Research Office-Durham, Grant DA-ARO-D-31-124-G1151. 
$f^{(n)}(z), n=1,2, \ldots$ The important operator $f \rightarrow f_{r}\left(f_{r}(z)=f(r z) / r, 0<r \leqq 1\right)$ gives rise to a function, order $f_{r}$. Theorem 3 shows that order $f_{r}$ is a monotone continuous function of $r, 0<r \leqq 1$. The use of the function order $f_{r}$ yields estimates on the $\mathscr{U}_{\beta^{-}}$ radius $(\beta \leqq \alpha)$ for the class $\mathscr{U}_{\alpha}$ (Theorem 4$)$, and leads to the interesting conclusion that the families $\mathscr{U}_{\alpha}$ are rotationally invariant (Theorem 5). Theorems 6 and 7 show that an analytic function must be transcendental entire if one imposes certain growth conditions on the $\mathscr{U}_{\beta}$-radii of the derivatives $f^{(n)}(z), n=0,1,2, \ldots$ Theorems 8 and 9 state the relations between the growth rate of the $\mathscr{U}_{\beta}$-radius of the derivatives and the order and type of an entire function. Theorems 10 and 11 show that control of the $\mathscr{U}_{\beta}$-radius on certain sequences of a function's derivatives is sufficient to force a function to be entire.

2. Preliminary linear invariant concepts. We begin by stating some of the basic definitions and results of the theory of linear invariant families of locally univalent analytic functions.

Let $\mathscr{J}$ be the set of all Möbius transformations of $D$ onto $D$, where $D=\{z:|z|<1\}$.

Pommerenke has defined [6] a family of functions of the form $f(z)=z+\cdots$ which are analytic and locally univalent $\left(f^{\prime}(z) \neq 0\right)$ in $D$ to be a linear invariant family if and only if for every $\phi(z) \in \mathscr{J}$, the function

$$
\Lambda_{\phi}[f(z)]=\frac{f(\phi(z))-f(\phi(0))}{\phi^{\prime}(0) \cdot f^{\prime}(\phi(0))}=z+\cdots
$$

is again a member of the family.

Linear invariant families exist in great profusion. The set of normalized univalent functions ( $p$-valent functions) which is denoted by $\mathscr{S}\left(\mathscr{S}_{p}\right)$ is linear invariant. Normalized convex functions, close-to-convex functions and functions with a fixed bound on the boundary rotation are all linear invariant families.

If $\mathscr{M}$ is a linear invariant family, then the order of $\mathscr{M}$ is defined as [6]

$$
\alpha=\sup \left\{\left|f^{\prime \prime}(0) / 2\right|: f(z) \in \mathscr{M}\right\} .
$$

We let $\mathscr{U}_{\alpha}$ denote the union of all linear invariant families of order at most $\alpha$. The (universal) family $\mathscr{U}_{\alpha}$ is itself linear invariant and has the following properties. If $\alpha<1$, then $\mathscr{U}_{\alpha}$ is empty [6, p. 117]. If $f(z)$ belongs to $\mathscr{U}_{\alpha}$, then we have the inequality

$$
\left|f^{\prime \prime}(0) / 2\right| \leqq \alpha
$$

which is of fundamental importance. The radius of convexity of $\mathscr{U}_{\alpha}$ is $\left[\alpha-\left(\alpha^{2}-1\right)^{1 / 2}\right]$ $[6$, p. 133].

If $f(z)$ is analytic and locally univalent in $D$, then we can consider the linear invariant family $\mathscr{M}(f)$ which it generates; namely,

$$
\mathscr{M}(f)=\left\{\Lambda_{\phi}[f(z)]: \phi(z) \in \mathscr{J}\right\} .
$$


We define the order of $f(z)$ to be the order of the linear invariant family generated by $f(z)$. As an aid in computing the order of $f(z)$, abbreviated order $f$, we have $[6$, p. 115]

$$
\operatorname{order} f=\sup _{z \in D}\left|-\bar{z}+\frac{\left(1-|z|^{2}\right)}{2} \frac{f^{\prime \prime}(z)}{f^{\prime}(z)}\right|=\sup \left|\frac{\Lambda_{\phi}[f(z)]^{\prime \prime}}{2}\right|_{z=0} \mid
$$

where the last supremum is taken over all $\phi(z)$ of the form $\phi(z)=(z+\zeta) /(1+\zeta z)$ with $|\zeta|<1$. For the ease of notation, we let

$$
t(z, f)=\left|-\bar{z}+\frac{\left(1-|z|^{2}\right)}{2} \frac{f^{\prime \prime}(z)}{f^{\prime}(z)}\right| .
$$

We have the following facts concerning the order of well-known families. The family $\mathscr{S}$ is contained in $\mathscr{U}_{2}$ [6, p. 115]. Close-to-convex functions of order $\beta$ are in $\mathscr{U}_{\beta+1}$ [7, p. 182]. Functions whose boundary rotation is bounded by $A$ are in $\mathscr{U}_{\alpha}$ where $\alpha=A / 2 \pi$. The family $\mathscr{U}_{1}$ is precisely the set of all normalized convex univalent functions $[6$, p. 134].

For each $\alpha>1, \quad \mathscr{U}_{\alpha}$ contains the function $(2 i \gamma)^{-1}\left([(1+z) /(1-z)]^{i \gamma}-1\right)$ $\left(\gamma=\left(\alpha^{2}-1\right)^{1 / 2}\right)$ which has order $\left(1+\gamma^{2}\right)^{1 / 2}$ and infinite valence [6, p. 128]. Thus the family $\mathscr{U}_{2}$, which contains $\mathscr{S}$ and for which many of the theorems of univalent function theory hold, is larger than $\mathscr{S}$ to the point of including functions of infinite valence.

\section{Admissible properties.}

Definition [10]. Let $f(z)$ be analytic in $D$ and possess a certain property $\mathscr{A}$ in $D$. The property $\mathscr{A}$ is called an admissible property if

(1) whenever $f(z)$ has $\mathscr{A}$, then $f^{\prime}(0) \neq 0$,

(2) whenever $f(z)$ has $\mathscr{A}$, then the function $F(z)=b f(z)+c$ has $\mathscr{A}$ for all $b, c$ complex numbers with $b \neq 0$.

If $\mathscr{A}$ is an admissible property, let $\mathscr{T}(\mathscr{A})$ be the set of all $f(z)=z+\cdots$ which are analytic in $D$ and for which $f^{(n)}(z)$ has $\mathscr{A}$ for all $n \geqq 0$. Let $A(\mathscr{A})=A$ $=\sup \left\{\left|a_{2}\right|: f \in \mathscr{T}(\mathscr{A})\right\}$.

Shah and Trimble prove the following basic theorem concerning $\mathscr{T}(\mathscr{A})$ :

THEOREM 1 [10]. If $\mathscr{A}$ is an admissible property and $A<\infty$, then, for any $f(z)$ $=z+\sum_{k=2}^{\infty} a_{k} z^{k}$ in $\mathscr{T}(\mathscr{A})$,

(1) $f(z)$ is an entire transcendental function of order 1 , and exponential type $\leqq 2 A$,

(2) $\left|a_{k}\right| \leqq(2 A)^{k-1} / k !, n=1,2, \ldots$,

(3) $|f(z)| \leqq(\exp \{2 A|z|\}-1) / 2 A, 0 \leqq|z|<\infty$,

(4) $\left|f^{(n)}(z)\right| \leqq(2 A)^{n-1} \exp (2 A|z|), 0 \leqq|z|<\infty, n \geqq 1$.

We say that $f$ has property $S$ if $f$ is univalent in $D$, and that $f$ has property $K$ if $f$ is univalent and $f(D)$ is a convex set. These are both admissible properties, and Shah and Trimble show that $\pi / 2 \leqq A(S)<1.7208$ and $\frac{1}{2} \leqq A(K)<.68379$. However, univalence and convexity of a mapping function are far stronger than being merely 
admissible properties; each of these properties can be used to generate linear invariant families. In fact we can apply Theorem 1 to the families $\mathscr{U}_{\alpha}$. We say that $f(z)$ has the property $\mathscr{U}(\alpha)$ if and only if $(f(z)-f(0)) / f^{\prime}(0)$ is in $\mathscr{U}_{\alpha}$.

THEOREM 2. The property $\mathscr{U}(\alpha)$ is admissible. Let $\mathscr{T}(\mathscr{U}(\alpha))$ be the set of all $f(z)$ $=z+\sum_{k=2}^{\infty} a_{k} z^{k}$ such that $f^{(n)}(z)$ has $\mathscr{U}(\alpha)$ for all $n \geqq 0$. Let

$$
A(\alpha)=\sup \left\{\left|a_{2}\right|: f \in \mathscr{T}(\mathscr{U}(\alpha))\right\} .
$$

Then $\left(\alpha+\left(\alpha^{2}-1\right)^{1 / 2}\right) / 2 \leqq A(\alpha) \leqq \alpha$. In particular as $\alpha \rightarrow \infty, A(\alpha)$ is arbitrarily close to $\alpha$.

Proof. It is trivial to verify that $\mathscr{U}(\alpha)$ is an admissible property. Furthermore since $f(z) \equiv(f(z)-f(0)) / f^{\prime}(0) \in \mathscr{U}_{\alpha}$ we have $\mathscr{T}(\mathscr{U}(\alpha)) \subset \mathscr{U}_{\alpha}$ and hence $A(\alpha) \leqq \alpha$.

We now examine the function $f(z)=\left(e^{a z}-1\right) / a$ where $a \geqq 1$. This function has the peculiar property that

$$
\left(f^{(n)}(z)-f^{(n)}(0)\right) / f^{(n+1)}(0) \equiv f(z)
$$

for all $n \geqq 0$. We now calculate the order of $f(z)$ using (2.3):

$$
\alpha=\sup _{z \in D}\left|-\bar{z}+\frac{\left(1-|z|^{2}\right)}{2} a\right|=\sup _{0 \leqq r<1}\left|r+\frac{\left(1-r^{2}\right)}{2} a\right|=\frac{1}{2}\left(a+\frac{1}{a}\right) .
$$

Thus $f(z)=\left(e^{a z}-1\right) / a$ is in $\mathscr{T}(\mathscr{U}(\alpha))$ where $\alpha=(a+1 / a) / 2$. The inequality $A(\alpha)$ $\geqq\left(\alpha+\left(\alpha^{2}-1\right)^{1 / 2}\right) / 2$ follo vs from the observation that $f^{\prime \prime}(0) / 2=a / 2$.

Since $\alpha \geqq A(\alpha) \geqq\left(\alpha+\left(\alpha^{2}-1\right)^{1 / 2}\right) / 2$, we see that

$$
|\alpha-A(\alpha)| \leqq 1 /\left[2\left(\alpha+\left(\alpha^{2}-1\right)^{1 / 2}\right)\right] \rightarrow 0 \quad \text { as } \alpha \rightarrow \infty .
$$

Thus asymptotically, $A(\alpha)=\alpha$. This concludes the proof of the theorem.

One can modify in various ways the notion that $f(z)$ has property $\mathscr{U}(\alpha)$. For example, we could say that $f(z)$ has property $\mathscr{A}$ if $(f(z)-f(0)) / f^{\prime}(0)$ is in $\mathscr{U}_{\alpha} \cap \mathscr{S}$, or $\mathscr{U}_{\alpha} \cap \mathscr{S}_{p}$ or $\mathscr{U}_{\alpha} \cap \mathscr{M}$ where $\mathscr{M}$ is any linear invariant family. The difficulty with each of these modifications is the determination of a suitable lower bound for $A(\alpha)$.

Theorem 2 shows that it is not the geometric property of univalence or convexity on the normalized derivatives of $f(z)$ that forces $f(z)$ to be an entire function of exponential type, rather it is the local univalence and the finite order of the family which is doing the work.

4. The $\mathscr{U}_{\beta}$-radius. Throughout the remainder of this paper the symbols $\alpha$ and $\beta$ will always be arbitrary real numbers $\geqq 1$.

The requirement of $\S 3$ that all of the normalized derivatives of a function be in $\mathscr{S}$ or $\mathscr{U}_{\alpha}$ is very restrictive. In order to force a function to be entire, Shah and Trimble found it sufficient to control the radius of univalence of the $n$th derivative.

For the families $\mathscr{U}_{\alpha}$, we have found that it is appropriate to replace the radius of univalence by a quantity that we call the $\mathscr{U}_{\beta}$-radius (defined below). Thus we proceed to develop the appropriate tools to apply this idea to the families $\mathscr{U}_{\alpha}$. 
We say that $f(z)$ has a radius of univalence $R$ at the origin, if the disc $D(0, R)$ is the largest disc centered at the origin in which $f(z)$ is univalent; equivalently, $R$ is the largest positive real number such that $[f(R z)-f(0)] /\left(R f^{\prime}(0)\right)$ is in $\mathscr{S}$. It is therefore natural to define the $\mathscr{U}_{\beta}$-radius of a function $f(z)$ as the largest positive real number $r$ such that

$$
(f(r z)-f(0)) / r f^{\prime}(0) \in \mathscr{U}_{\beta} .
$$

In particular, we note that if $(f(r z)-f(0)) / r f^{\prime}(0)$ is in $\mathscr{U}_{1}$, then $(f(r z)-f(0)) / r f^{\prime}(0)$ is convex [6, p. 134]. Thus the $\mathscr{U}_{1}$-radius of a function is the same as the radius of convexity of a function. Since $\mathscr{S} \subset \mathscr{U}_{2}$, the $\mathscr{U}_{2}$-radius of a function is never less than its radius of univalence. Since it is the order property of a function which is often of importance in applications and not the geometrical property of univalence (see [2]), the $\mathscr{U}_{\beta}$-radius is a meaningful quantity.

We confine our investigations to the $\mathscr{U}_{\beta}$-radius for the class $\mathscr{U}_{\alpha}$. The $\mathscr{U}_{\beta}$-radius for the class $\mathscr{U}_{\alpha}$ is the largest positive real number $r$ such that for all $f$ in $\mathscr{U}_{\alpha}$ we have $(f(r z)-f(0)) / r f^{\prime}(0)$ in $\mathscr{U}_{\beta}$.

For the function

$$
f(z)=\frac{1}{2 \alpha}\left(\left(\frac{1+z}{1-z}\right)^{\alpha}-1\right), \quad \alpha \geqq 1,
$$

Pommerenke has shown that order $f=\alpha[6, \mathrm{p} .117]$, and therefore the $\mathscr{U}_{\beta}$-radius for $\mathscr{U}_{\alpha}$ is precisely 1 if $\beta \geqq \alpha$.

LEMMA 1. An analytic function $f(z)$ has $a \mathscr{U}_{\beta}$-radius $\geqq R$ if and only if

$$
\sup _{z \in D}\left|-\bar{z}+\frac{\left(1-|z|^{2}\right)}{2} R \frac{f^{\prime \prime}(R z)}{f^{\prime}(R z)}\right| \leqq \beta .
$$

Proof. This follows immediately from (4.1) and (2.3).

If $f(z) \in \mathscr{U}_{\alpha}$, then $(f(r z)-f(0)) / r f^{\prime}(0)$ is just $f(r z) / r$. This latter operation is of great importance to univalent function theory since in many cases results for the whole class $\mathscr{S}$ can be derived from the more tractable subclass of functions univalent and analytic on $\bar{D}$.

For functions in $\mathscr{U}_{\alpha}$, one should expect an intimate relation between the $\mathscr{U}_{\beta^{-}}$ radius and the order of the function $f_{r}(z)=f(r z) / r$ for fixed $r, 0<r \leqq 1$. We therefore examine how the order of $f_{r}(z)$ changes with $r$.

LEMMA 2. If $f(z)$ has order $\alpha$, then order $f_{r} \leqq(\alpha-1) r+1$ for $0<r \leqq 1$. In particular if $\alpha>1$, and $0<r<1$, then order $f_{r}<\alpha$.

Proof. We fix $r(0<r<1)$ and we fix $f(z)$ with order $\alpha$. It suffices (see (2.3)) to show for any $\phi(z)=(z+\zeta) /(1+\zeta z),|\zeta|<1$, that if $g(z)=\Lambda_{\phi}\left[f_{r}(z)\right]$ then $\left|g^{\prime \prime}(0) / 2\right|$ $\leqq(\alpha-1) r+1$.

We let

$$
\phi^{*}(z)=r \phi(z), \quad \omega(z)=\frac{z+\phi^{*}(0)}{1+\overline{\phi^{*}(0) z}} \quad \text { and } \quad \chi(z)=\frac{\phi^{*}(z)-\phi^{*}(0)}{1-\overline{\phi^{*}(0)} \phi^{*}(z)}
$$


It is easy to check that

$$
g(z)=\Lambda_{\phi}\left[f_{r}(z)\right]=\Lambda_{\phi}[f(z)]=\Lambda_{\omega \circ x}[f(z)]=\Lambda_{x}\left[\Lambda_{\omega}[f(z)]\right]=\Lambda_{x}[h(z)]
$$

where $h(z)=\Lambda_{\omega}[f(z)] \in \mathscr{U}_{\alpha}$ (see $[6$, p. 118]).

Easy calculations show that

$$
g^{\prime \prime}(0) / 2=\frac{1}{2} \chi^{\prime \prime}(0) / \chi^{\prime}(0)+\frac{1}{2} h^{\prime \prime}(0) \chi^{\prime}(0)
$$

and

and hence

$$
\chi(z)=r z\left(1-|\zeta|^{2}\right) /\left(\left(1-r^{2}\right) \xi z+1-r^{2}|\zeta|^{2}\right)
$$

$$
\begin{gathered}
\left|\chi^{\prime}(0)\right|=r\left(1-|\zeta|^{2}\right) /\left(1-r^{2}|\zeta|^{2}\right) \leqq r \\
\left|\chi^{\prime \prime}(0) / \chi^{\prime}(0)\right| /\left[2\left(1-\left|\chi^{\prime}(0)\right|\right)\right]=(1+r)|\zeta| /\left(1+r|\zeta|^{2}\right) \leqq 1 .
\end{gathered}
$$

Since $h(z) \in \mathscr{U}_{\alpha}$ we have

$$
\left|h^{\prime \prime}(0) / 2\right| \leqq \alpha .
$$

Putting (4.2), (4.4) and (4.5) together we find that

$$
\begin{aligned}
\frac{1}{2}\left|g^{\prime \prime}(0)\right| & \leqq \frac{1}{2}\left|\chi^{\prime \prime}(0) / \chi^{\prime}(0)\right|+\frac{1}{2}\left|h^{\prime \prime}(0)\right|\left|\chi^{\prime}(0)\right| \\
& \leqq 1-\left|\chi^{\prime}(0)\right|+\left|\chi^{\prime}(0)\right| \alpha \leqq(\alpha-1) r+1 .
\end{aligned}
$$

If $\alpha>1$, then we note that equality can hold in (4.6) if and only if $\left|\chi^{\prime}(0)\right|=r$ which is the case if and only if $r=1$ (see (4.3)). This concludes the proof of the Lemma.

THEOREM 3. Let the order of $f(z)$ be $\alpha$. Then order $f_{r}$ is a nondecreasing continuous function of $r$ for $r \in(0,1]$. Furthermore, order $f_{r}$ is strictly increasing for $r \geqq$ the radius of convexity of $f(z)$. Finally,

$$
\operatorname{order} f_{r} \leqq 1+(\alpha-1) r
$$

and if $\alpha>1$, equality holds in (4.7) if and only if $r=1$.

Proof. We begin by showing that $\operatorname{order} f_{r}$ is monotone nondecreasing. If $0<r<s$ we let $G(z)=f_{s}(z)$. Then $f_{r}(z)=G_{r / s}(z)$ and by Lemma 2

$$
\operatorname{order} f_{r} \leqq\left(\operatorname{order} f_{s}-1\right) r / s+1 \leqq \operatorname{order} f_{s} .
$$

If $r>$ radius of convexity of $f(z)$, we know that order $f_{r}>1$ and hence in (4.8) we have strict inequality; that is, order $f_{r}$ is strictly increasing for $r>$ radius of convexity of $f(z)$.

We now show that $\operatorname{order} f_{r}$ is a left continuous function for $r \in(0,1)$. We let $M=\max \left|f^{\prime \prime}(r z) / f^{\prime}(r z)\right|$ for $z \in \bar{D}$. Clearly $M$ is finite since $r \in(0,1)$ is fixed. For $\varepsilon>0$, we choose $\delta_{1}$ and $\delta_{2}$ such that $\delta_{1} M<\varepsilon / 4$, and if $s$ is in $0<r-\delta_{2}<s<r$ then

$$
\max _{z \in \bar{D}}\left|\frac{f^{\prime \prime}(r z)}{f^{\prime}(r z)}-\frac{f^{\prime \prime}(s z)}{f^{\prime}(s z)}\right|<\varepsilon / 4 \text {. }
$$


If we let $\delta=\min \left(\delta_{1}, \delta_{2}\right)$ then

$$
\begin{aligned}
& \left|\left(-\bar{z}+\frac{\left(1-|z|^{2}\right)}{2} r \frac{f^{\prime \prime}(r z)}{f^{\prime}(r z)}\right)-\left(-\bar{z}+\frac{\left(1-|z|^{2}\right)}{2} s \frac{f^{\prime \prime}(s z)}{f^{\prime}(s z)}\right)\right| \\
& \leqq \frac{\left(1-|z|^{2}\right)}{2}\left[|r-s|\left|\frac{f^{\prime \prime}(r z)}{f^{\prime}(r z)}\right|+s\left|\frac{f^{\prime \prime}(r z)}{f^{\prime}(r z)}-\frac{f^{\prime \prime}(s z)}{f^{\prime}(s z)}\right|\right]<\frac{\varepsilon}{2}
\end{aligned}
$$

for all $s$ in $0<r-\delta<s<r$. But by Lemma 2, order $f_{s} \leqq \operatorname{order} f_{r}$. We now choose $z_{0}$ so that $t\left(z_{0}, f_{r}\right)=\operatorname{order} f_{r}$ and apply (4.9) to show that order $f_{s} \geqq \operatorname{order} f_{r}-\varepsilon$. Thus, for $r-\delta<s<r$ we have that order $f_{r} \geqq \operatorname{order} f_{s} \geqq \operatorname{order} f_{r}-\varepsilon$, hence order $f_{r}$ is left continuous. The proof that order $f_{r}$ is right continuous is identical except for choosing $z_{0}$ so that $t\left(z_{0}, f_{s}\right)=\operatorname{order} f_{s}$.

We are using Theorem 4.3 of [3], with an obvious gloss that order $f_{r}>1$, to guarantee that there is a $z_{0}$ in $D$ such that $t\left(z_{0}, f_{r}\right)=\operatorname{order} f_{r}$.

For continuity at $r=1$, we choose $z_{0}$ so that $t\left(z_{0}, f\right)$ is within $\varepsilon / 2$ of order $f$, and then choose $s$ so that $t\left(z_{0}, f_{s}\right)$ is within $\varepsilon / 2$ of $t\left(z_{0}, f\right)$. Thus

$$
\text { order } f \leqq t\left(z_{0}, f_{s}\right)+\varepsilon \leqq \operatorname{order} f_{s}+\varepsilon \leqq \operatorname{order} f+\varepsilon .
$$

Since order $f_{t}$ is increasing in $t$, we have shown from (4.10) that $\operatorname{order} f-\varepsilon$ $\leqq \operatorname{order} f_{t} \leqq \operatorname{order} f$ for $s<t<1$ which shows that order $f_{r}$ is continuous on the left at $r=1$.

Finally (4.7) follows directly from Lemma 2 which concludes the proof of Theorem 3.

THEOREM 4. If $R_{\beta}$ is the $\mathscr{U}_{\beta}$-radius for the class $\mathscr{U}_{\alpha}$, then

$$
\max \left[\left[\alpha-\left(\alpha^{2}-1\right)^{1 / 2}\right],(\beta-1) /(\alpha-1)\right] \leqq R_{\beta} \leqq\left[\beta+\left(\beta^{2}-1\right)^{1 / 2}\right]\left[\alpha-\left(\alpha^{2}-1\right)^{1 / 2}\right] .
$$

Proof. Since the families $\mathscr{U}_{\alpha}$ are nested, it is clear that the $\mathscr{U}_{\beta}$-radius for any class of functions is never less than the $\mathscr{U}_{1}$-radius (radius of convexity) of the class. But the radius of convexity of $\mathscr{U}_{\alpha}$ is $\left[\alpha-\left(\alpha^{2}-1\right)^{1 / 2}\right]$. Furthermore for

$$
r=(\beta-1) /(\alpha-1)
$$

we obtain from Theorem 3 that

$$
\sup _{z \in D}\left|-\bar{z}+\frac{\left(1-|z|^{2}\right)}{2} r \frac{f^{\prime \prime}(r z)}{f^{\prime}(r z)}\right| \leqq(\alpha-1) r+1 \equiv \beta .
$$

Thus $R_{\beta} \geqq r=(\beta-1) /(\alpha-1)$ which implies

$$
\max \left[\left(\alpha-\left(\alpha^{2}-1\right)^{1 / 2}\right),(\beta-1) /(\alpha-1)\right] \leqq R_{\beta} .
$$

To establish the right-hand side of (4.11), we consider the function $f(z)$ $=\left(e^{a z}-1\right) / a \in \mathscr{U}_{\alpha}$ for $a=\alpha+\left(\alpha^{2}-1\right)^{1 / 2}$. We use Lemma 1 and for

$$
R=\left(\beta+\left(\beta^{2}-1\right)^{1 / 2}\right)\left(\alpha-\left(\alpha^{2}-1\right)^{1 / 2}\right),
$$


we find that

$$
\begin{aligned}
\sup _{z \in D}\left|-\bar{z}+\frac{\left(1-|z|^{2}\right)}{2} R \frac{f^{\prime \prime}(R z)}{f^{\prime}(R z)}\right| & =\sup _{z \in D}\left|-\bar{z}+\frac{\left(1-|z|^{2}\right)}{2}\left(\beta+\left(\beta^{2}-1\right)^{1 / 2}\right)\right| \\
& =\sup _{0 \leqq r<1}\left[r+\frac{\left(1-r^{2}\right)}{2}\left(\beta+\left(\beta^{2}-1\right)^{1 / 2}\right)\right]=\beta,
\end{aligned}
$$

since the maximum of $r+\left(1-r^{2}\right)\left(\beta+\left(\beta^{2}-1\right)^{1 / 2}\right) / 2$ occurs at $r=\left(\beta+\left(\beta^{2}-1\right)^{1 / 2}\right)^{-1}$. It is easy to check that no larger value of $R$ is possible and therefore the $\mathscr{U}_{\beta}$-radius of $f(z)=\left(e^{a z}-1\right) / a$ is precisely $\left(\beta+\left(\beta^{2}-1\right)^{1 / 2}\right)\left(\alpha-\left(\alpha^{2}-1\right)^{1 / 2}\right)$. This establishes the upper bound for $R_{\beta}$ and completes the proof of the theorem.

We remark that the theorem yields the precise $\mathscr{U}_{\beta}$-radius in the case of $\beta=1$ or $\beta=\alpha$.

The preceding proof enables us to prove as an easy corollary the following known result $[6$, p. 133].

COROLlaRY 1. The radius of convexity of $\mathscr{U}_{\alpha}$ is precisely $\left(\alpha-\left(\alpha^{2}-1\right)^{1 / 2}\right)$.

Proof. For any $f(z) \in \mathscr{U}_{\alpha}$, we obtain from (2.3) that

$$
\operatorname{Re}\left(1+z f^{\prime \prime} \mid f^{\prime}(z)\right) \geqq\left(|z|^{2}-2 \alpha|z|+1\right) /\left(1-|z|^{2}\right) .
$$

Thus all $f(z)$ in $\mathscr{U}_{\alpha}$ are convex in $|z| \leqq\left(\alpha-\left(\alpha^{2}-1\right)^{1 / 2}\right)$. The previous proof explicitly displays a function in $\mathscr{U}_{\alpha}$ for which this number cannot be exceeded.

One notes for the family $\mathscr{U}_{2}$ that both the Köbe function $f(z)=z /(1-z)^{2}$ and the function $g(z)=\left[e^{(2+\sqrt{ } 3) z}-1\right] /(2+\sqrt{ } 3)$ have the smallest possible radius of convexity, namely $2-\sqrt{ } 3$, and both have order precisely 2 . Yet the two functions are in no manner equivalent. The function $f(z)$ is analytic and univalent in $|z|<1$ (rational and bivalent in $|z|<\infty)$; the function $g(z)$ is entire and has infinite valence in $|z|<\infty$. This nonuniqueness of extremal functions in $\mathscr{U}_{\alpha}$ contrasts sharply with the usual situation for elementary extremal problems in the class $\mathscr{S}$. Similar nonuniqueness examples arise for the problem of max $\left|a_{2}\right|$ in $\mathscr{U}_{\alpha}$ or even in $\mathscr{U}_{\alpha} \cap \mathscr{S}$.

We note that since a starlike function is univalent, Corollary 2.5 of Pommerenke [6, p. 134] assures us that for the class $\mathscr{U}_{\alpha}$, the radius of univalence, $R_{u}$, of $\mathscr{U}_{\alpha}$ satisfies

$$
1 / \alpha \leqq R_{u} \leqq \tanh \left(\pi / 2\left(\alpha^{2}-1\right)^{1 / 2}\right)
$$

We now establish a fundamental lemma that relates the radius of univalence of a function $f(z)$ with the $\mathscr{U}_{\beta}$-radius of $f(z)$.

LEMMA 3. If $f(z)$ is analytic in $|z|<1$ and has $\mathscr{U}_{\beta}$-radius $r_{\beta}$, then its radius of univalence, $r_{u}$, satisfies the inequality:

$$
\beta^{-1} \cdot r_{\beta} \leqq r_{u} \leqq r_{\beta}(2+\sqrt{ } 3) .
$$

Proof. Since $f(z)$ has $\mathscr{U}_{\beta}$-radius $r_{\beta}$, we know that

$$
F(z)=\left(f\left(r_{\beta} z\right)-f(0)\right) /\left(r_{\beta} \cdot f^{\prime}(0)\right) \in \mathscr{U}_{\beta} .
$$


Hence by (4.12), the radius of univalence of $F(z)$ is no less than $1 / \beta$. From (4.14), it follows that the radius of univalence of $f(z)$ is no less than $r_{\beta} / \beta$ which establishes the left-hand side of (4.13).

We shall examine two cases to establish the right-hand side. If $2 \leqq \beta<\infty$, then clearly $r_{u} \leqq r_{2} \leqq r_{\beta} \leqq r_{\beta}(2+\sqrt{ } 3)$, where $r_{2}$ is the $\mathscr{U}_{2}$-radius of $f(z)$. However, if $1 \leqq \beta \leqq 2$ we can only state that $r_{u} \leqq r_{2}$. Since

$$
G(z)=\left(f\left(r_{2} z\right)-f(0)\right) /\left(r_{2} \cdot f^{\prime}(0)\right) \in \mathscr{U}_{2},
$$

Theorem 4 guarantees that $G(z)$ has a $\mathscr{U}_{\beta}$-radius $r(G)_{\beta} \geqq(2+\sqrt{ } 3)^{-1}$. Therefore

$$
\frac{G\left(r(G)_{\beta} z\right)-G(0)}{r(G)_{\beta} \cdot G^{\prime}(0)} \equiv \frac{f\left(r_{2} \cdot r(G)_{\beta} \cdot z\right)-f(0)}{r_{2} \cdot r(G)_{B} \cdot f^{\prime}(0)} \in \mathscr{U}_{\beta} .
$$

Hence, by definition of $r_{\beta}$, we must have $r_{\beta} \geqq r_{2} \cdot r(G)_{\beta}$ and consequently

$$
r_{u} \leqq r_{2} \leqq r_{\beta}\left[r(G)_{\beta}\right]^{-1} \leqq r_{\beta}(2+\sqrt{ } 3)
$$

which concludes the lemma.

We say that a family of functions $\mathscr{F}$ is purely rotationally invariant if whenever $f(z)$ is in $\mathscr{F}$, then $e^{-i \theta} f\left(e^{i \theta} z\right)$ is also in $\mathscr{F}$ for all $0 \leqq \theta \leqq 2 \pi$. A family of functions $\mathscr{F}$ is contractionally invariant if whenever $f(z)$ is in $\mathscr{F}$, then $f(r z) / r$ is also in $\mathscr{F}$ for all $0<r \leqq 1$. Starlike functions, convex functions, close-to-convex functions and $\mathscr{S}$ itself are examples of purely rotationally invariant families and contractionally invariant families. Kirwan has defined a family of functions $\mathscr{F}$ to be rotationally invariant if it is both purely rotationally and contractionally invariant. Rotational invariance is a property of great value in the study of extremal problems by variational methods [4]. Thus it is of some interest that the family $\mathscr{U}_{\alpha}$ is not only linearly invariant but also rotationally invariant.

THEOREM 5. The family $\mathscr{U}_{\alpha}$ is a rotationally invariant family for all $\alpha \geqq 1$.

Proof. Use of (2.1) with $\phi(z)=e^{i \theta} z$ shows that $\mathscr{U}_{\alpha}$ is purely rotationally invariant. Theorem 3 shows that if $f(z)$ in $\mathscr{U}_{\alpha}$, then the order of $f_{r}(z)$ is no greater than $\alpha$, hence $f_{r}(z)$ is in $\mathscr{U}_{\alpha}$ for all $0<r \leqq 1$. Therefore $\mathscr{U}_{\alpha}$ is also contractionally invariant.

For our purposes, the rotational invariance of the family $\mathscr{U}_{\alpha}$ shows that the $\mathscr{U}_{\beta}$-radius has the important property of being a monotone function of $\beta$.

5. Derivatives with variable $\mathscr{U}_{\beta}$-radius. We first note that if $f(z)$ is an analytic function in $D$ then $f^{(n)}(z)$ will have a positive $\mathscr{U}_{\beta}$-radius $r_{n}(\beta)=r_{n}$ if and only if $f^{(n+1)}(0)=(n+1) ! a_{n+1} \neq 0$. Thus the eventual nonvanishing of the coefficients $a_{k}$ of $f(z)=\sum_{k=0}^{\infty} a_{k} z^{k}$ will have strong implications for any question concerning the limiting behavior of the $\mathscr{U}_{\beta}$-radii of the derivatives of $f(z)$. We therefore introduce the following definitions due to Shah and Trimble [9].

Let $f(z)$ be an analytic function in $|z|<R$ ( $R$ may be infinite). We say that $f(z)$ has property $A$ at $N$ if there is a nonnegative integer $N$ such that for all $n \geqq N$, $a_{n+1} \neq 0$; equivalently, $r_{n}(\beta)>0$. 
The behavior of $\left|a_{n}\right| a_{n+1} \mid$ restricts the radius of convergence $R$ of $f(z)$. Therefore, we shall say that $f(z)$ has property $B$ at $N$ if there is a positive integer $N$ such that $\left\{\left|a_{n-1} / a_{n}\right|\right\}_{n=N}^{\infty}$ is a positive nondecreasing sequence. Clearly if $f(z)$ has property $B$ then it must also have property $A$. Furthermore, if $f(z)$ has property $B$ then it has radius of convergence $R=\lim \inf \left|a_{n-1} / a_{n}\right|=\lim \sup \left|a_{n-1} / a_{n}\right|$.

THEOREM 6. Let $f(z)=\sum_{k=0}^{\infty} a_{k} z^{k}$. Let $R$ denote the radius of convergence of $f(z)$ and let $r_{n}(\beta)=r_{n}$ denote the $\mathscr{U}_{\beta}$-radius of $f^{(n)}(z), n=0,1,2, \ldots$ Then

$$
\liminf _{n \rightarrow \infty} n r_{n} \leqq 2 \beta R,
$$

$$
R(\log 2)(2+\sqrt{ } 3)^{-1} \leqq \limsup _{n \rightarrow \infty} n r_{n} .
$$

If $f(z)$ has property $A$ at $N$, then

$$
\liminf _{n \rightarrow \infty}\left[n\left(r_{N} \cdots r_{n}\right)^{1 / n}\right] \leqq 2 \beta e R .
$$

If $f(z)$ has property $B$ at $N$, then

$$
R(\log 2)(2+\sqrt{ } 3)^{-1} \leqq \liminf _{n \rightarrow \infty} n r_{n} \leqq \limsup _{n \rightarrow \infty} n r_{n} \leqq 2 \beta R .
$$

Proof. If $f(z)$ does not have property $A$, then $r_{n}=0$ for infinitely many $n$ and (5.1) is trivial. On the other hand, if $f(z)$ has $A$ at $N$, then

$$
F_{n}(z)=\left[f^{(n)}\left(r_{n} z\right)-f^{(n)}(0)\right] / r_{n} f^{(n+1)}(0) \in \mathscr{U}_{\beta}
$$

for all $n \geqq N$. Thus by our fundamental relation $(2.2),\left|F_{n}^{\prime \prime}(0) / 2\right| \leqq \beta$ and therefore

$$
(n+2) r_{n} \leqq 2 \beta\left|a_{n+1} / a_{n+2}\right| \text {. }
$$

However, $\lim \inf \left|a_{n} / a_{n+1}\right| \leqq R$ and this establishes (5.1). If $f(z)$ has property $B$, then we actually have $\lim \sup \left|a_{n} / a_{n+1}\right|=R$ which, with (5.5), establishes the right-hand side of (5.4).

Using (5.5), an induction argument shows that for all $k \geqq N+2$ we have

$$
\left|a_{k}\right| \leqq(2 \beta)^{k-N-1}\left|a_{N+1}\right|(N+1) ! /\left(r_{N} \cdots r_{k-2}\right) k ! \text {. }
$$

Stirling's estimate shows that $(k !)^{1 / k}$ approaches $k / e$ and therefore

$$
1 / R=\limsup _{k \rightarrow \infty}\left|a_{k}\right|^{1 / k} \leqq 2 \beta e / \lim \inf \left[k\left(r_{N} \cdots r_{k-2}\right)^{1 / k}\right]
$$

or

$$
\liminf _{k \rightarrow \infty}\left[k\left(r_{N} \cdots r_{k-2}\right)^{1 / k}\right] \leqq 2 \beta e R .
$$

We note that (5.3) is obvious from (5.6) if $R=\infty$. If $R$ is finite, then $r_{k}<R<\infty$ and lim sup $\left(r_{k-1} r_{k}\right)^{1 / k} \leqq 1$. This fact and (5.6) allow us to conclude that (5.3) is true for all cases.

If $f(z)$ is an arbitrary analytic function in $|z|<R$, Shah and Trimble have shown $\left[9\right.$, p. 315] that for any $0<r<R$, there is a sequence $\{n(p)\}_{p=1}^{\infty}$ of positive integers 
such that $r \cdot x(n(p)) \leqq R_{n(p)} \cdot n(p)$ where $R_{n(p)}$ is the radius of univalence of $f^{(n(p))}(z)$ and $x(n)=n\left[1-2^{-1 /(n+2)}\right]$. Therefore by Lemma 3 we have that

$$
r \cdot x(n(p)) \leqq R_{n(p)} \cdot n(p) \leqq(2+\sqrt{ } 3) r(\beta)_{n(p)} \cdot n(p)
$$

where $r(\beta)_{n(p)}$ is the $\mathscr{U}_{\beta}$-radius of $f^{(n(p))}(z)$. Since $\lim _{n \rightarrow \infty} n\left[1-2^{-1 /(n+2)}\right]=\log 2$, we obtain (5.2) from (5.7) by taking the limit supremum.

If $f(z)$ has property $B$, then we modify the above proof in conjunction with $\left[9\right.$, p. 315] by letting $r_{n}=\left|a_{n-1} / a_{n}\right| \leqq R$ and obtain

$$
x(n(p)) \cdot r_{n(p)} \leqq n(p) \cdot R_{n(p)} \leqq(2+\sqrt{ } 3) n(p) \cdot r(\beta)_{n(p)} .
$$

Therefore since $\lim _{n \rightarrow \infty} n\left[1-2^{-1 /(n+2)}\right] r_{n}=R \log 2$, we obtain the left-hand side of (5.4). This concludes the proof of the theorem.

COROLlaRY 2. Let $r_{n}(\beta)=r_{n}$ denote the $\mathscr{U}_{\beta}$-radius of the nth derivative. If $\lim _{n \rightarrow \infty} n r_{n}=\infty$ then $f(z)$ is an entire transcendental function. If $f(z)$ is transcendental entire, then $\lim _{n \rightarrow \infty} n r_{n}=\infty$. If $f(z)$ is transcendental entire with property $B$, then $\lim \sup _{n \rightarrow \infty} n \cdot r_{n}=\infty$.

THEOREM 7. Let $f(z)$ be analytic in $D$. If there is a positive integer $N$ such that for all $n \geqq N$

$$
F_{n}(z)=\left[f^{(n)}(z)-f^{(n)}(0)\right] / f^{(n+1)}(0) \in \mathscr{U}_{k n^{1-\varepsilon}}
$$

where $k>0$ and $\varepsilon>0\left(k N^{1-\varepsilon} \geqq 1\right)$, then $f(z)$ is a transcendental entire function.

Proof. If $F_{n}(z) \in \mathscr{U}_{k n^{1-\varepsilon}}$ for $n \geqq N$, then $F_{n}(z)$ has a $\mathscr{U}_{\beta}$-radius greater than or equal to $(B-1) /\left(k n^{1-\varepsilon}-1\right)$ by Theorem 4 and this implies that $\lim _{i n f} \inf _{n \rightarrow \infty} n r_{n}=\infty$. Thus, by Corollary $2, F_{N}(z)$, hence $f(z)$ itself, must be an entire transcendental function.

We note that the following special cases yield known results:

(a) If $\varepsilon=1, k=2$, we have a generalization of part of Theorem 1 [8].

(b) If $\varepsilon=1, k=1$, we have part of Theorem 2 [10].

(c) If $\varepsilon=1, k \geqq 1$, we have part of the conclusion of Theorem 2 of this paper.

The converses to the first and second parts of Corollary 2 are false. Indeed with (5.5) and (4.13) one can make the necessary modifications in Shah and Trimble's work [9] to generalize their counterexamples to the present setting.

6. Entire functions and the $\mathscr{U}_{\beta}$-radius of the derivatives. In this section we state the relations between the order and type of an entire function $f(z)$ and the $\mathscr{U}_{\beta}$-radius of $f^{(n)}(z)$. The advantage of the present setting over that involving the radius of univalence of $f^{(n)}(z)$ is two-fold: (1) All of the previous estimates are exactly the same for the much larger class $\mathscr{U}_{2}$. (2) Since the $\mathscr{U}_{\beta}$-radius involves an explicit algebraic expression, the determination of the $\mathscr{U}_{\beta}$-radius is often easier than that of the radius of univalence. Furthermore, the natural setting for the proofs seems to be that of the general area of linear invariant families of locally univalent functions.

We begin by proving a technical lemma. 
LEMMA 4. Let $0 \leqq J<\infty$. If $\left\{\lambda_{k}\right\}_{k=1}^{\infty}$ is a sequence of real numbers for which there is an $N$ such that for all $k \geqq N$ we have $\lambda_{k}>0$, then

$$
e^{J-1} \liminf _{k \rightarrow \infty} k^{J-1} \lambda_{k}^{J} \leqq \liminf _{k \rightarrow \infty} k^{J-1}\left(\lambda_{N} \cdots \lambda_{k}\right)^{J / k} .
$$

Proof. We let $M=e^{J-1} \lim \inf _{k \rightarrow \infty} k^{J-1} \lambda_{k}^{J}$ and assume that $0<M<\infty$ since (6.1) is certainly true if $M=0$. Corresponding to $\varepsilon>0$, there is an $N^{\prime}=N^{\prime}(\varepsilon)>N$ such that $\lambda_{k}^{J} k^{J-1}>(M-\varepsilon) e^{1-J}$ for all $k \geqq N^{\prime}$. Hence for $k \geqq N^{\prime}$,

$$
\begin{aligned}
k^{J-1}\left(\lambda_{N} \cdots \lambda_{k}\right)^{J / k} & \geqq k^{J-1}\left(\lambda_{N} \cdots \lambda_{N^{\prime}-1}\right)^{J / k} \cdot\left(\prod_{j=N^{\prime}}^{k} \frac{(M-\varepsilon) e^{1-J}}{j^{J-1}}\right)^{1 / k} \\
= & \left(\lambda_{N} \cdots \lambda_{N^{\prime}-1}\right)^{J / k}\left((M-\varepsilon) e^{1-J}\right)^{\left(k-N^{\prime}+1\right) / k}\left(\left(N^{\prime}-1\right) !\right)^{(J-1) / k}\left(k^{k} / k !\right)^{(J-1) / k} .
\end{aligned}
$$

Using Stirling's estimate and taking the limit infimum, we observe that the righthand side of $(6.1)$ is $\geqq(M-\varepsilon)$. Since $\varepsilon$ is arbitrary, we have established (6.1) in the case $0 \leqq M<\infty$. The case of $M=\infty$ proceeds exactly as above.

This lemma is needed in Theorem 8 to prove the left-hand inequality of (6.5) where we let $\lambda_{k}$ denote the $\mathscr{U}_{\beta}$-radius of a function with property $A$.

We shall omit all proofs of our results in this section, since all of these proofs can be obtained from obvious modifications of the work of Shah and Trimble [9] with the application of (5.5) and (4.13) in the spirit of Theorem 6 and Theorem 10. Throughout this section we shall let $\Lambda$ be the order and $\lambda$ the lower order of $f(z)$. If $0<\Lambda<\infty$, let $T$ denote the type and $t$ the lower type of $f(z)$. (For definitions of these terms see [1].) Let $r_{n}(\beta)=r_{n}$ denote the $\mathscr{U}_{\beta}$-radius of $f^{(n)}(z)$ where $\beta$ is a fixed but arbitrary number $\geqq 1$.

Lemma 5. Let $f(z)=\sum_{n=0}^{\infty} a_{n} z^{n}$ be defined in $|z|<R$ ( $R$ may be infinite). Let $v(r)$ denote its central index. For $n=1,2, \ldots$ we let $x(n)=n\left(1-2^{-1 /(n+2)}\right)$. If $0<r<R$ and $v(r) \geqq 2$, then

$$
r \cdot x(v(r)-1)<(v(r)-1) \cdot(2+\sqrt{ } 3) r_{v(r)-1} .
$$

THEOREM 8. Let $f(z)=\sum_{k=0}^{\infty} a_{n} z^{n}$ be an entire transcendental function. Let $\delta=\lim _{\inf _{r \rightarrow \infty}} v(r) / r$. Then

$$
\begin{gathered}
\liminf _{n \rightarrow \infty} \frac{\log \left(\max \left[1, n r_{n}\right]\right)}{\log n} \leqq \frac{1}{\Lambda}, \\
\frac{1-\lambda}{\lambda} \leqq \operatorname{iimsup}_{n \rightarrow \infty}\left[\frac{\log r_{n}}{\log n}\right],
\end{gathered}
$$$$
(\log 2)(2+\sqrt{ } 3)^{-1} \leqq \limsup _{n \rightarrow \infty} r_{n} \text {. }
$$

If $0<\Lambda<\infty$ and $f(z)$ has property $A$ at $N$, then

$$
e^{\Lambda-1} \liminf _{n \rightarrow \infty} n^{\Lambda-1} r_{n}^{\Lambda} \leqq \liminf _{n \rightarrow \infty} n^{\Lambda-1}\left(r_{N} \cdots r_{n}\right)^{\Lambda / n} \leqq \frac{(2 \beta)^{\Lambda} e^{\Lambda-1}}{\Lambda T} .
$$


In any case,

$$
\liminf _{n \rightarrow \infty} n^{\Lambda-1} r_{n}^{\Lambda} \leqq(2 \beta)^{\Lambda} / \Lambda T
$$

Corollary 3. If $\lambda<1$, then lim $\sup _{n \rightarrow \infty} r_{n}=\infty$. If $\Lambda=1$ then

$$
\frac{(\log 2)(2+\sqrt{ } 3)^{-1}}{t} \leqq \frac{(\log 2)(2+\sqrt{ } 3)^{-1}}{\delta} \leqq \limsup _{n \rightarrow \infty} r_{n}
$$

In particular, if $\delta=0$, then $\lim \sup _{n \rightarrow \infty} r_{n}=\infty$.

Corollary 4. If $\Lambda>1$, then $\liminf _{n \rightarrow \infty} r_{n}=0$. If $\Lambda=1$, then $(2 \beta) T^{-1}$ $\geqq \lim \inf _{n \rightarrow \infty} r_{n}$. In particular, if $\Lambda=1$ and $T=\infty$, then $\lim \inf _{n \rightarrow \infty} r_{n}=0$.

We shall see in the remaining results that if $f(z)$ has property $B$, then we can obtain information about $\lim r_{n}$ rather than just $\lim \inf r_{n}$ or $\lim \sup r_{n}$.

THEOREM 9. Let $f(z)$ be an entire function with property $B$ at $N$. Let $\gamma=\lim \sup _{r \rightarrow \infty} v(r) / r$. Then

$$
\begin{aligned}
& \liminf _{n \rightarrow \infty} {\left[\frac{\log r_{n}}{\log n}\right]=\frac{1-\Lambda}{\Lambda} \leqq \frac{1-\lambda}{\lambda}=\limsup _{n \rightarrow \infty}\left[\frac{\log r_{n}}{\log n}\right], } \\
& \frac{(\log 2)(2+\sqrt{ } 3)^{-1}}{\gamma} \leqq \liminf _{n \rightarrow \infty} r_{n} \leqq \frac{2 \beta}{\gamma} \\
& \frac{(\log 2)(2+\sqrt{ } 3)^{-1}}{\delta} \leqq \liminf _{n \rightarrow \infty} r_{n} \leqq \frac{2 \beta}{\delta}
\end{aligned}
$$

If $0<\Lambda<\infty$, then

$$
\left(\frac{\log 2}{2+\sqrt{ } 3}\right)^{\Lambda} \leqq \Lambda t e^{1-\Lambda} \limsup _{n \rightarrow \infty}\left(r_{N} \cdots r_{n}\right)^{\Lambda / n} n^{\Lambda-1} \leqq(2 \beta)^{\Lambda}
$$

and

$$
\left(\frac{\log 2}{2+\sqrt{ } 3}\right)^{\Lambda} \leqq \Lambda T e^{1-\Lambda} \liminf _{n \rightarrow \infty}\left(r_{N} \cdots r_{n}\right)^{\Lambda / n} n^{\Lambda-1} \leqq(2 \beta)^{\Lambda}
$$

COROLlaRY 5. Let $f(z)$ be an entire function with property $B$.

(i) If $\Lambda<1$, then $\lim _{n \rightarrow \infty} r_{n}=\infty$.

(ii) If $\lambda>1$, then $\lim _{n \rightarrow \infty} r_{n}=0$.

(iii) If $\Lambda=1$, then $\lim _{n \rightarrow \infty} r_{n}=0$ if and only if $T=0$. If $\lim _{n \rightarrow \infty} r_{n}=0$, then $t=\infty$.

7. Functions with infinitely many $r_{n}=0$. If $f(z)$ does not have property $A$, then the conclusion of Theorem 6 that $\lim _{\inf _{n \rightarrow \infty}} n r_{n} \leqq 2 \beta R$ is of course trivial, since $r_{n}=0$ for infinitely many $n$. Thus Theorem 6 gives us no information for odd functions, i.e. $f(z)=\sum_{n=0}^{\infty} a_{2 n+1} z^{2 n+1}$. Therefore we shall assume that there is a sequence of derivatives $\left\{f^{(n(p))}(z)\right\}_{p=1}^{\infty}$ for which $r_{n(p)}>0$.

THEOREM 10. Let $\{n(p)\}_{p=1}^{\infty}$ be a strictly increasing sequence of positive integers. Let $f(z)=\sum_{n=0}^{\infty} a_{n} z^{n}$ have a radius of convergence $R \geqq 1$. Let $f^{(n(p))}(z)$ have $\mathscr{U}_{\beta}$-radius 
$r_{n(p)} \geqq 1$. Then

$$
\liminf _{p \rightarrow \infty}\left(r_{n(1)} \cdots r_{n(p)}\right)^{1 / n(p)} \liminf _{p \rightarrow \infty}(n(1) \cdots n(p))^{1 / n(p)} \leqq 2 \beta R .
$$

Proof. Since

$$
F_{n(p)}(z)=\frac{f^{(n(p))}\left(r_{n(p)} z\right)-f^{(n(p))}(0)}{r_{n(p)} \cdot f^{(n(p)+1)}(0)} \in \mathscr{U}_{\beta},
$$

by our fundamental relation (2.2) we have the inequality

$$
\left|f^{(n(p)+2)}(0) \cdot r_{n(p)} / 2 \cdot f^{(n(p)+1)}(0)\right| \leqq \beta
$$

or

$$
|a[n(p)+2]| \leqq \frac{2 \beta|a[n(p)+1]|}{r_{n(p)} \cdot[n(p)+2]}<\frac{2 \beta|a[n(p)+1]|}{r_{n(p)} \cdot n(p)}
$$

where for typographical reasons we let $a[k]$ denote $a_{k}$, the $k$ th coefficient of $f(z)$.

As noted before, the radius of univalence of any function in $\mathscr{U}_{\beta}$ is at least $1 / \beta$, hence $\beta F_{n(p)}(z / \beta)$ is in $\mathscr{S}$. By Littlewood's estimate [5, p. 218], the $k$ th coefficient of $F_{n(p)}(z)$ is bounded by $e \cdot k$. Therefore,

$$
\left|\beta \cdot r_{n(p)}^{k-1} \cdot f^{(n(p)+k)}(0)\right| f^{(n(p)+1)}(0) \cdot k ! \mid<e k
$$

or

$$
|a[n(p)+k]| \leqq \frac{(e k) k !(n(p)+1) ! a[n(p)+1]}{\beta\left(r_{n(p)}\right)^{k-1}(n(p)+k) !} .
$$

Since $(e k) k !(n(p)+1) ! / \beta\left(r_{n(p)}\right)^{k-1}(n(p)+k) !$ is decreasing in $k$, for $p \geqq 2$, it is easy to show that for all $p \geqq 27$ and all $k \geqq 3$

$$
\frac{e k \cdot k !(n(p)+1) !}{\beta\left(r_{n(p)}\right)^{k-1}(n(p)+k) !}<\frac{2 \beta}{r_{n(p)} \cdot n(p)} .
$$

An application of (7.3) to (7.2) and remembering (7.1) yields

$$
|a[n(p)+k]|<2 \beta|a[n(p)+1]| /\left(r_{n(p)} \cdot n(p)\right)
$$

for $p \geqq 27$ and all $k \geqq 2$. By an induction argument, for $p \geqq 27$ and $k$ such that $n(p)$ $+1<n(p)+k \leqq n(p+1)+1$, we obtain

$$
|a[n(p)+k]|<\frac{(2 \beta)^{p-26}|a[n(27)+1]|}{\left(\prod_{k=27}^{p} n(k) r_{n(k)}\right)}=\frac{(2 \beta)^{p} \cdot A}{\left(\prod_{k=1}^{p} n(k) r_{n(k)}\right)},
$$

where $A$ is the obvious constant. Therefore

$$
\limsup _{k \rightarrow \infty}\left|a_{k}\right|^{1 / k} \leqq \frac{\lim \sup _{p \rightarrow \infty}(2 \beta)^{p / n(p)}}{\lim \inf _{p \rightarrow \infty}\left(n_{1} \cdots n_{p}\right)^{1 / n(p)} \lim \inf _{p \rightarrow \infty}\left(r_{n(1)} \cdots r_{n(p)}\right)^{1 / n(p)}}
$$

However, $\lim \sup _{k \rightarrow \infty}\left|a_{k}\right|^{1+k}=1 / R$ and thus

$$
\liminf _{p \rightarrow \infty}\left(n_{1} \cdots n_{p}\right)^{1 / n(p)} \liminf _{p \rightarrow \infty}\left(r_{n(1)} \cdots r_{n(p)}\right)^{1 / n(p)} \leqq R \limsup _{p \rightarrow \infty}(2 \beta)^{p / n(p)} \leqq 2 \beta R .
$$


Actually Theorem 10 and Shah and Trimble's original Theorem 3 [10] are both too strong for easy applications.

THEOREM 11. If there is a strictly increasing sequence of positive integers $\{n(p)\}_{p=1}^{\infty}$ such that

(a) $\lim \inf _{p \rightarrow \infty}(n(1) \cdots n(p))^{1 / n(p)}=\infty$ and

(b) for a function $f(z)$, analytic in a neighborhood of $0, \lim _{\inf } \rightarrow \infty r_{n(p)}>0$, then $f(z)$ is an entire transcendental function.

Proof. We let $\lim \inf _{p \rightarrow \infty} r_{n(p)}=a$. Then by our fundamental Lemma 3, we know that $\lim \inf R_{n(p)} \geqq a / \beta$, where $R_{n(p)}$ is the radius of univalence of $f^{(n(p))}(z)$. If we consider the function $F(z)=f(a z / \beta)$, then $F(z)$ is analytic in $D$ and $F^{(n(p))}(z)$ has radius of univalence, hence $\mathscr{U}_{2}$ radius, no less than 1 . Thus by Theorem 10 , we obtain the result that $F(z)$ is entire, and hence that $f(z)$ itself is an entire transcendental function.

Shah and Trimble's corollary [10, p. 454] holds in the following more general setting.

CoROllary 6. If $n(p+1)-n(p)=o(\log (n(p)+1))$, and $\lim \inf r_{n(p)}>0$, then $f(z)$ is entire. In particular, if $f(z)$ is an odd function and $\lim \inf r_{2 n+1}>0$, then $f(z)$ is entire.

I wish to thank Professor John Pfaltzgraff for suggesting this topic, his proof of the critical Lemma 2, and his valuable suggestions in the preparation of this paper.

\section{BIBLIOGRAPHY}

1. R. P. Boas, Entire functions, Academic Press, New York, 1954. MR 16, 914.

2. D. M. Campbell, Majorization and subordination theory for locally univalent functions (to appear).

3. D. M. Campbell, J. A. Cima and J. A. Pfaltzgraff, Linear spaces and linear invariant families of locally univalent analytic functions, Manuscripta Math. 4 (1971), 1-30.

4. W. E. Kirwan, A note on extremal problems for certain classes of analytic functions, Proc. Amer. Math. Soc. 17 (1966), 1028-1030. MR 34 \#2854.

5. Z. Nehari, Conformal mapping, McGraw-Hill, New York, 1952. MR 13, 640.

6. Ch. Pommerenke, Linear-invariante Familien analytischer Funktionen. I, Math. Ann. 155 (1964), 108-154. MR 29 \#6007.

7. - On close-to-convex analytic functions, Trans. Amer. Math. Soc. 114 (1965), 176-186. MR 30 \#4920.

8. S. M. Shah and S. Y. Trimble, Univalent functions with univalent derivatives, Bull. Amer. Math. Soc. 75 (1969), 153-157. MR 39 \#433.

9. —_ Univalent functions with univalent derivatives. II, Trans. Amer. Math. Soc. 144 (1969), 313-320. MR 40 \#2841.

10. - Univalent functions with univalent derivatives. III, J. Math. Mech. 19 (1969/70), 451-460. MR 40 \#4438.

University of North Carolina, Chapel Hill, North Carolina 27514 